\title{
HADRON CORRELATORS IN THE DECONFINED PHASE ${ }^{a}$
}

\author{
F. KARSCH \\ Fakultät für Physik, Universität Bielefeld, D-33615 Bielefeld, Germany \\ E-mail:karsch@physik.uni-bielefeld.de \\ M.G. MUSTAFA \\ Theoretical Nuclear Physics Division, Saha Institute of Nuclear Physics, 1/AF \\ Bidhan Nagar, Calcutta - 700 064, India \\ E-mail: mustafa@tnp.saha.ernet.in \\ M.H. THOMA \\ Theory Division, CERN, CH-1211 Geneva 23, Switzerland \\ E-mail: markus.thoma@cern.ch
}

\begin{abstract}
Temporal meson correlators and their spectral functions are calculated in the deconfined phase using the hard thermal loop resummation technique. The spectral functions exhibit strong medium effects coming from the hard thermal loop approximation for the quark propagator. The correlators, on the other hand, do not differ significantly from free correlators, for which bare quark propagators are used. This is in contrast to lattice calculations showing a clear deviation from the free correlations functions.
\end{abstract}

\section{Introduction}

QCD Lattice calculations have been used successfully for studying hadron properties at zero temperature. At finite temperature there is a sudden change of these properties as soon as the critical temperature $T_{c}$ for the deconfinement transition has been reached. However, in particular in the pseudo-scalar channel the mesonic correlation functions differ clearly from free correlators, describing freely propagating bare quarks, even above $T_{c} \mathbb{E}$. These deviations could be caused either by bound states of quarks above $T_{c}$ or by in-medium modifications of the collective quark modes in the QGP. In order to investigate the origin of the non-trivial correlations we calculate temporal meson correlations functions using the hard thermal loop (HTL) resummation techniquel. This improved perturbation theory for QCD at high temperatures $\left(T \gg T_{c}\right)$ takes into account important medium effects of the QGP such as effective, temperature dependent quark masses and Landau damping $\mathrm{B}$. Therefore it is interesting to investigate to what extent the HTL approximation for the mesonic correlators is able to explain the lattice results. In particular tem-

${ }^{a}$ Talk given at the "Strong and Electro-Weak Matter" Conference (14-17 June 2000 Marseille) 
poral correlators and their spectral functions, which we will consider in the following exclusively, yield interesting information about hadronic properties at finite temperature.

\section{Meson Correlators and Spectral Functions}

Meson correlators are defined as expectation values

$$
G_{M}(\tau, \vec{x})=\left\langle J_{M}(\tau, \vec{x}) J_{M}^{\dagger}(0, \overrightarrow{0})\right\rangle
$$

of meson currents $J_{M}(\tau, \vec{x})=\bar{q}(\tau, \vec{x}) \Gamma_{M} q(\tau, \vec{x})$ in Euclidean time $\tau=i t \epsilon[0,1 / T]$. Here $q(x)$ denotes the quark wave function and $\Gamma_{M}=1, \gamma_{5}, \gamma_{\mu}, \gamma_{\mu} \gamma_{5}$ the quarkmeson vertex corresponding to the mesonic channel (scalar, pseudo-scalar, vector, pseudo-vector) under consideration.

The temporal correlator, defined as the limit $\vec{x}=0$ of the correlation function, is related to the correlation function in momentum space by

$$
G_{M}(\tau, \vec{x}=0)=T \sum_{n=-\infty}^{\infty} \mathrm{e}^{-i \omega_{n} \tau} \chi_{M}\left(\omega_{n}, \vec{p}=0\right)
$$

where $\omega_{n}=2 \pi n T$ are the bosonic Matsubara frequencies.

An interesting quantity which we will study in the following is the spectral function of the mesonic correlator. As we will see, the spectral function contains much more information about medium effects in the QGP than the correlator itself. It is defined as

$$
\chi_{M}\left(\omega_{n}, \vec{p}\right)=-\int_{-\infty}^{\infty} \mathrm{d} \omega \frac{\sigma_{M}(\omega, \vec{p})}{i \omega_{n}-\omega+i \epsilon} .
$$

Using (2) and (3) the temporal correlator can be expressed by

$$
G_{M}(\tau)=\int_{0}^{\infty} \mathrm{d} \omega \sigma_{M}(\omega, \vec{p}=0) \frac{\cosh (\omega(\tau-1 / 2 T))}{\sinh (\omega / 2 T)} .
$$

\section{Free Correlators}

The free mesonic correlators follow from the one-loop self-energy diagram of Fig.1(a) containing bare quark propagators. In momentum space the correlation function reads

$$
\chi_{M}(\omega, \vec{p})=2 N_{c} T \sum_{n} \int \frac{\mathrm{d}^{3} k}{(2 \pi)^{3}} \operatorname{Tr}\left[\Gamma_{M} S_{F}\left(k_{0}, \vec{k}\right) \Gamma_{M}^{\dagger} S_{F}^{\dagger}\left(\omega-k_{0}, \vec{p}-\vec{k}\right)\right],
$$


(a)

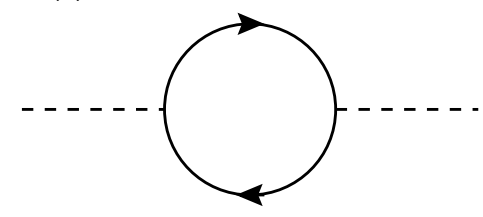

(b)

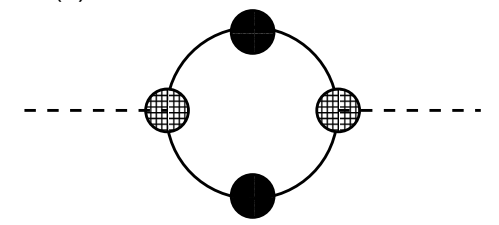

Figure 1: Meson correlators for free quarks (a) and in the HTL approximation (b).

where $k_{0}=i(2 n+1) \pi T$ are the fermionic Matsubara frequencies. This leads to the following expression for the spectral functions of the free temporal correlator

$$
\sigma_{M}^{\text {free }}(\omega)=\frac{N_{c}}{4 \pi^{2}} \theta(\omega-2 m) \omega^{2} \tanh \left(\frac{\omega}{4 T}\right) \sqrt{1-\left(\frac{2 m}{\omega}\right)^{2}}\left[a_{M}+\left(\frac{2 m}{\omega}\right)^{2} b_{M}\right]
$$

where $\sigma_{v}=\sigma_{v}^{\mu}$ in the (pseudo-)vector case. The coefficients $\left(a_{M}, b_{M}\right)$ are given by $(-1,1),(1,0),(2,1)$, and $(-2,3)$ in the scalar, pseudo-scalar, vector, and pseudo-vector case, respectively. For massless bare quarks, $m=0,\left|\sigma_{p s}\right|=$ $\left|\sigma_{s}\right|$ and $\left|\sigma_{p v}\right|=\left|\sigma_{v}\right|$ hold reflecting chiral symmetry. For $m=0$ analytic expressions can be derived 6 for the temporal correlator from (6) using (4). For large real times $t \rightarrow \infty$ they reduce to $G_{M}(t) \sim \exp (-\mu t)$, with the screening mass $\mu=2 \pi T$. The same screening mass has also been found for spatial correlators at large distances 4 .

\section{HTL Approximation}

Now we want to consider the HTL approximation for the temporal correlator and its spectral function using HTL resummed propagators and vertices as shown in Fig.1(b). In this way important medium effects of the high temperature plasma, namely effective temperature dependent quark masses and Landau damping are taken into account. The HTL quark propagator in the imaginary time formalism can be written as

$$
S_{F}^{\mathrm{HTL}}\left(k_{0}, \vec{k}\right)=-\left(\gamma_{0} k_{0}-\vec{\gamma} \cdot \vec{k}\right) \int_{0}^{1 / T} \mathrm{~d} \tau \mathrm{e}^{k_{0} \tau} \int_{-\infty}^{\infty} \mathrm{d} \omega \rho_{\mathrm{HTL}}(\omega, \vec{k})\left[1-n_{F}(\omega)\right] \mathrm{e}^{-\omega \tau},
$$

where $n_{F}(\omega)=1 /(1+\exp (\omega / T))$ is the Fermi distribution. It is convenient to decompose the spectral function of the HTL quark propagator according to 
its helicity eigenstates

$$
\rho_{\mathrm{HTL}}(\omega, \vec{k})=\frac{1}{2} \rho_{+}(\omega, k)\left(\gamma_{0}-i \hat{k} \cdot \vec{\gamma}\right)+\frac{1}{2} \rho_{-}(\omega, k)\left(\gamma_{0}+i \hat{k} \cdot \vec{\gamma}\right),
$$

where

$$
\rho_{ \pm}(\omega, k)=\frac{\omega^{2}-k^{2}}{2 m_{q}^{2}}\left[\delta\left(\omega-\omega_{ \pm}\right)+\delta\left(\omega+\omega_{\mp}\right)\right]+\beta_{ \pm}(\omega, k) \theta\left(k^{2}-\omega^{2}\right) .
$$

Here the first term of (9) comes from the poles of the HTL propagator with the in-medium quark dispersion relation $\omega_{ \pm}(k)$. This dispersion relation exhibits two branches, of which the upper one $\left(\omega_{+}\right)$corresponds to a collective quark mode and the lower one $\left(\omega_{-}\right)$to a plasmino having a negative ratio of helicity to chirality and being absent in vacuum 0 . The plasmino dispersion shows a minimum at non-vanishing momentum as a general property of massless fermions in relativistic plasmas 6 . The cut contribution, coming from the imaginary part of the quark self energy which describes Landau damping, reads

$$
\beta_{ \pm}(\omega, k)=-\frac{m_{q}^{2}}{2} \frac{ \pm \omega-k}{\left[k(-\omega \pm k)+m_{q}^{2}\left( \pm 1-\frac{ \pm \omega-k}{2 k} \ln \frac{k+\omega}{k-\omega}\right)\right]^{2}+\left[\frac{\pi}{2} m_{q}^{2} \frac{ \pm \omega-k}{k}\right]^{2}}
$$

where the effective quark mass in the HTL approximation is given by $m_{q}=$ $g(T) T / \sqrt{6}$.

According to the above decomposition of the quark propagator in a pole and a cut contribution, the spectral function of the temporal correlator following from Fig.1(b) can be written as a sum of a pole-pole, pole-cut, and a cut-cut term, $\sigma^{\mathrm{HTL}}(\omega)=\sigma^{\mathrm{pp}}(\omega)+\sigma^{\mathrm{pc}}(\omega)+\sigma^{\mathrm{cc}}(\omega)$. The most interesting term is the pole-pole contribution, for which we obtain for the pseudo-scalar spectral function

$$
\begin{aligned}
& \sigma_{\mathrm{ps}}^{\mathrm{pp}}(\omega)=\frac{N_{c}}{2 \pi^{2} m_{q}^{4}}\left(\mathrm{e}^{\omega / T}-1\right)\left[n_{F}^{2}\left(\omega_{+}\left(k_{1}\right)\right) \frac{\left(\omega_{+}^{2}\left(k_{1}\right)-k_{1}^{2}\right)^{2} k_{1}^{2}}{2\left|\omega_{+}^{\prime}\left(k_{1}\right)\right|}\right. \\
& +2 \sum_{i=1}^{2} n_{F}\left(\omega_{+}\left(k_{2}^{i}\right)\right)\left[1-n_{F}\left(\omega_{-}\left(k_{2}^{i}\right)\right)\right] \frac{\left(\omega_{-}^{2}\left(k_{2}^{i}\right)-\left(k_{2}^{i}\right)^{2}\right)\left(\omega_{+}^{2}\left(k_{2}^{i}\right)-\left(k_{2}^{i}\right)^{2}\right)\left(k_{2}^{i}\right)^{2}}{\left|\omega_{+}^{\prime}\left(k_{2}^{i}\right)-\omega_{-}^{\prime}\left(k_{2}^{i}\right)\right|} \\
& \left.+\sum_{i=1}^{2} n_{F}^{2}\left(\omega_{-}\left(k_{3}^{i}\right)\right) \frac{\left(\omega_{-}^{2}\left(k_{3}^{i}\right)-\left(k_{3}^{i}\right)^{2}\right)^{2}\left(k_{3}^{i}\right)^{2}}{2\left|\omega_{-}^{\prime}\left(k_{3}^{i}\right)\right|}\right] .
\end{aligned}
$$

Here we have neglected the HTL vertex correction in Fig.1(b) since in the case of the (pseudo-)scalar correlator it leads to higher order corrections only, as 

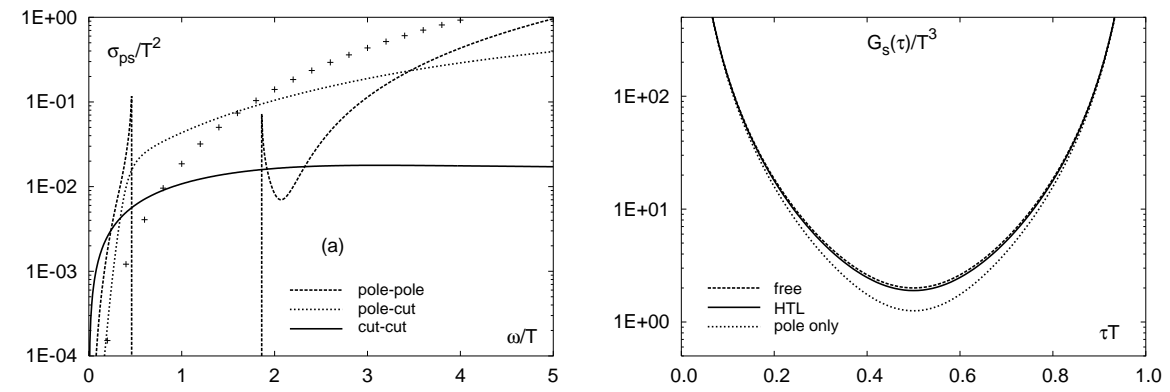

Figure 2: Left panel: The pole-pole, pole-cut and cut-cut contributions to the pseudo-scalar spectral function for $m_{q} / T=1$. The crosses show the free meson spectral function. Right panel: The thermal pseudo-scalar meson correlation function in the HTL approximation for $m_{q} / T=1$. The curves show the complete thermal correlator (middle line), the correlator constructed from $\sigma_{\mathrm{s}}^{\mathrm{pp}}$ only (lower line) and the free thermal correlator (upper line).

in Yukawa theory 0 . The momenta $k_{n}^{i}$ are determined from the zeros of the equations $\omega-2 \omega_{+}\left(k_{1}\right)=0, \omega-\omega_{+}\left(k_{2}^{i}\right)+\omega_{-}\left(k_{2}^{i}\right)=0$, and $\omega-2 \omega_{-}\left(k_{3}^{i}\right)=0$. Due to the minimum in the plasmino dispersion two solutions for $k_{2}^{i}$ and $k_{3}^{i}$ $(i=1,2)$ are possible. The first term in (11) describes the annihilation of collective quarks, the second one the transition from the upper to the lower branch, and the last one the annihilation of plasminos. The transition process starts at zero energy and continues until the maximum difference $\omega=0.47 m_{q}$ between the two branches at $k_{2}=1.18 m_{q}$. At this point a Van Hove singularity is encountered due to the vanishing denominator $\omega_{+}^{\prime}\left(k_{2}\right)-\omega_{-}^{\prime}\left(k_{2}\right)=0$, where the prime denotes the derivative with respect to $k$. The plasmino annihilation starts at $\omega=1.86 m_{q}$ with another Van Hove singularity corresponding to the minimum of the plasmino branch at $k_{3}=0.41 m_{q}$, where $\omega_{-}^{\prime}\left(k_{3}\right)=0$. This contribution falls off rapidly due to the exponentially suppressed spectral strength of the plasmino mode for large energies, where only the first process, quark-antiquark annihilation starting at $\omega=2 m_{q}$, contributes. The pole-cut and cut-cut contributions, on the other hand, are smooth functions of $\omega$. The individual contributions to the spectral function of the temporal pion correlator are shown in the left panel of Fig.2 for $m_{q} / T=1$.

Surprisingly the temporal correlator following from integrating over $\omega$ according to (4) is similar to the free one in spite of the significant structures showing up in the spectral function (right panel of Fig.2). There is a cancellation of the (due to the effective quark mass) reduced pole-pole contribution compared to the free correlator and the additional pole-cut and cut-cut con- 
tributions to a large extent.

A similar behavior can be observed for the temporal vector correlator $\mathrm{B}$. In this case a HTL quark-meson vertex has to be considered as in the case of the closely related soft dilepton production rate 6 . The pole-pole contribution to the spectral function contains again Van Hove singularities at the same energies as in the pseudo-scalar case. However, the cut-cut contribution diverges now for small energies like $\sigma_{v}{ }^{\mathrm{cc}}(\omega) \sim 1 / \omega$ leading to a singular result for the temporal vector correlator after integrating over $\omega$.

\section{Conclusions}

Lattice calculations of meson correlators in the deconfined phase show a clear deviation from the free correlation functions in particular in the pseudo-scalar channel. The long range behavior of free correlators in time and space can be described by a screening mass $\mu=2 \pi T$ corresponding to the propagation of two bare quarks. Using HTL resummed quark propagators instead of bare ones, medium effects, such as thermal quark masses and Landau damping, due to interactions with the thermal particles of the QGP are taken into account. This leads to sharp structures (Van Hove singularities, energy gap) in the spectral functions of the temporal correlators. However, the correlators following from their spectral functions by an energy integration are close to the free correlators due to a partial cancellation of the pole-pole and the cut contributions in the HTL approximation. In other words, HTL medium effects appear not to be sufficient to explain the lattice results. This supports speculations about bound states or other non-perturbative effects in the QGP close to the critical temperature.

\section{Acknowledgments}

FK has been supported by the TMR network ERBFMRX-CT-970122 and the DFG under grant Ka 1198/4-1. MGM would like to acknowledge support from $\mathrm{AvH}$ Foundation as part of this work was initiated during his stay at the

University of Giessen as Humboldt Fellow. MHT has been supportd by the DFG as Heisenberg Fellow.

\section{References}

1. G. Boyd, S. Gupta, F. Karsch and E. Laermann, Z. Phys. C 64, 331 (1994).

2. E. Braaten and R.D. Pisarski, Nucl. Phys. B 337, 569 (1990). 
3. M. Le Bellac, Thermal Field Theory (Cambridge University Press, Cambridge, 1996).

4. W. Florkowski and B.L. Friman, Z. Phys. A 347, 271 (1994).

5. E. Braaten, R.D. Pisarski and T. C. Yuan, Phys. Rev. Lett. 64, 2242 (1990).

6. A. Peshier and M.H. Thoma, Phys. Rev. Lett. 84, 841 (2000).

7. M.H. Thoma, Z. Phys. C 66, 491 (1995).

8. F. Karsch, M.G. Mustafa, and M.H.Thoma, hep-ph/0007093. 